\title{
The Dynamics of Plant-Mediated Sediment Oxygenation in Spartina anglica Rhizospheres-a Planar Optode Study
}

\author{
Ketil Koop-Jakobsen • Frank Wenzhöfer
}

Received: 22 August 2013 / Revised: 24 June 2014 / Accepted: 10 July 2014 / Published online: 1 August 2014

(C) The Author(s) 2014. This article is published with open access at Springerlink.com

\begin{abstract}
Belowground sediment oxygenation in rhizospheres of wetland plants promotes nutrient uptake, serve as protection against toxic reduced compounds and play an important role in wetland nutrient cycling. The presence of $\sim 1.5$-mm-wide oxic zones around roots of the intertidal marsh grass Spartina anglica was demonstrated below the sediment surface using planar optode technology recording $2 \mathrm{D}$ images of the sediment oxygen distribution. Oxic root zones were restricted to the root tips stretching up to $16 \mathrm{~mm}$ along the roots with an oxygen concentration up to $85 \mu \mathrm{mol} \mathrm{L}{ }^{-1}$ detected at the root surface. Radial oxygen loss across the root surface ranged from 250 to $300 \mathrm{nmol} \mathrm{m}^{-2} \mathrm{~s}^{-1}$, which is comparable to other wetland plants. During air exposure of the aboveground biomass, atmospheric oxygen was the primary source for belowground oxygen transport, and light availability only had a minor effect on the belowground sediment oxygenation. During inundations completely submerging the aboveground biomass cutting off access to atmospheric oxygen, oxic root zones diminished significantly in the light and were completely eliminated in darkness. Within the time frame of a normal tidal inundation $(\sim 1.5 \mathrm{~h})$, photosynthetic oxygen production maintained the presence of oxic root zones in light, whereas oxic root zones were eliminated within $1 \mathrm{~h}$ in darkness. The results show that the sediment oxygenation in Spartina anglica rhizospheres is temporally dynamic as well as spatially variable along the roots.
\end{abstract}

Communicated by Marianne Holmer

K. Koop-Jakobsen · F. Wenzhöfer

HGF MPG Joint Research Group for Deep Sea Ecology and

Technology, Max Planck Institute for Marine Microbiology,

Celsiusstrasse 1, 28359 Bremen, Germany

K. Koop-Jakobsen $(\square)$

MARUM - Center for Marine Environmental Sciences, University

of Bremen, Leobener Str., 28359 Bremen, Germany

e-mail: kjakobsen@marum.de
Keywords Spartina anglica · Sediment oxygenation · Radial oxygen loss · Oxic microzones · Planar optode $\cdot$ Salt marsh . Wetlands

\section{Introduction}

Spartina anglica has well-developed aerenchyma tissue facilitating a rapid supply of oxygen from aboveground sources to the belowground roots and rhizomes, where it may leak across the rhizodermis and oxygenate the surrounding sediment, a process also known as radial oxygen loss (ROL) (Armstrong and Wright 1975). Teal and Kanwisher (1966) described oxygen translocation in the closely related Spartina alterniflora more than four decades ago and also suggested that the capacity for belowground oxygen transport may exceed the respiratory demand resulting in oxygen leakage across the root surface oxygenating the surrounding sediment. Later field observations of precipitated oxidized iron minerals on the surface of Spartina roots indirectly confirmed the excretion of oxygen across the rhizodermis to the surrounding sediment (Giblin and Howarth 1984; Mendelssohn and Postek 1982). Subsequently, various studies have investigated oxygen leakage from the roots of different species of Spartina with differing results; Lee (2003) found significant oxygen release from the roots of $S$. anglica, whereas Howes and Teal (1994) found no measurable oxygen loss through the roots in the closely related $S$. alterniflora. Studies by Maricle and Lee (2002, 2007) revealed that the oxygen transport capacity differs significantly among different species of Spartina with S. anglica having a markedly high oxygen transport capacity. The plant-mediated belowground sediment oxygenation, which is common in many wetland plants (Brix 1994) and also in seagrasses (Sand-Jensen et al. 1982) improves the plants ability to inhabit waterlogged and anoxic sediments (Lee 2003; Maricle and Lee 2002). Sediment oxidation may 
act as a mechanism to reduce the impact of reduced toxic compounds, especially $\mathrm{H}_{2} \mathrm{~S}$, accumulating in the otherwise permanently anoxic sediment/soil surrounding the roots (Lee 1999; Pezeshki 2001) as well as improving nutrient uptake (Bradley and Morris 1990; Lai et al. 2012).

S. anglica thrives in low marshes in a wide variety of substrates, including clays, fine silts, organic muds, sands and shingle, and is tolerant to regular tidal inundations with oceanic salinity (Gray and Marshall, A. Raybould 1991). S. anglica colonizes frequently inundated bare mudflats and competitively excludes native wetland species in low marshes (Nehring and Hesse 2008). S. anglica is successfully spreading in many wetlands and coastlines around the world from New Zealand to the North American west coast and is in many places considered an invading species (Nehring and $\mathrm{H}$. Adsersen 2006; Nehring and Hesse 2008). The high oxygen transport capacity of $S$. anglica is considered an important factor for its ability to inhabit bare mudflats and to outcompete native species in the low marsh (Maricle and Lee 2007).

Measuring oxygen directly in sediments surrounding roots and rhizomes is difficult without physically disturbing the sediment composition and damaging root tissue, and consequently, most studies of oxygen transport and leakage in Spartina spp. are conducted as hydroponic studies (Howes and Teal 1994; Maricle and Lee 2002; Maricle and Lee 2007; Teal and Kanwisher 1966) quantitatively describing the transport of oxygen through the plants, but leaving little information of the actual oxygenation of the sediment surrounding the plant roots. In 2002, Holmer et al. demonstrated the presence of distinct 2.5-mm-wide oxic zones around S. anglica roots using oxygen microelectrode profiling, but the extent of oxic zones inside the rhizosphere and their spatial distribution and temporal dynamics are still largely unknown.

Planar optode technology facilitates recording of oxygen distribution in sediments and soils as two-dimensional images (Glud et al. 1996; Holst et al. 1998) and is increasingly being used for investigations of plant-mediated sediment oxygenation at depth in waterlogged rhizospheres (Blossfeld 2013; Blossfeld and Gansert 2012; Gansert and Blossfeld 2008). Even so, its use in wetlands and shallow waters is still limited to very few species including Juncus spp. (Blossfeld et al. 2011), Zostera marina (Frederiksen and Glud 2006; Jensen et al. 2005) and Carex appressa (Minett et al. 2013). In this study, planar optodes were used to investigate plant-mediated sediment oxygenation around the roots of the intertidal marsh grass $S$. anglica, visualizing the presence of oxic root zones at depth in the sediment.

Oxygen translocated to the sediment via roots and rhizomes originates from the atmosphere and photosynthetic oxygen production. Consequently, belowground sediment oxygenation can be affected by tidal inundations cutting off access to atmospheric oxygen, in which case the oxygen transport is dependent on oxygen supply from the surrounding water, as well as internal photosynthetic oxygen production controlled by light availability. Furthermore, recent studies have demonstrated that the presence of gas films on hydrophobic surfaces of leaves of $S$. anglica can improve oxygen exchange and internal oxygen transport during tidal inundations (Winkel et al. 2011). In this study, we investigated the effect of light availability and tidal flooding on belowground sediment oxygenation in the laboratory monitoring oxic zones around roots of $S$. anglica under different light regimes and during air exposure and submergence of the aboveground biomass.

\section{Methods and Material}

\section{S. anglica Cultures}

The investigations of belowground sediment oxygenation by $S$. anglica were conducted on vegetation samples collected in the field and cultured in the laboratory for 8 months. The samples were collected on the German Wadden Sea Island, Sylt, in a location $\left(54^{\circ} 59^{\prime} 13.08^{\prime \prime} \mathrm{N} ; 8^{\circ} 22^{\prime} 10.50^{\prime \prime} \mathrm{E}\right)$, where $S$. anglica is growing as monoculture swards in intertidal sandy permeable sediments. A block of the $S$. anglica vegetation $(20 \mathrm{~cm} \times 20 \mathrm{~cm} \times 20 \mathrm{~cm})$ was dug out and brought to the laboratory, where the sediment was washed away from the dense network of roots and rhizomes. Smaller samples consisting of several interconnected shoots were carefully separated and replanted in 20-L plastic aquaria in sediment collected in the field. The replanted samples were placed inside a $400-\mathrm{L}$ storage tank and cultured at $15{ }^{\circ} \mathrm{C}$ under artificial lighting $\left(150 \mu \mathrm{mol}\right.$ photons $\mathrm{m}^{-2} \mathrm{~s}^{-1}$ photosynthetically active radiation (PAR)) with a daily cycle of 16-h light and 8-h darkness. These light conditions are lower than the field conditions, and the vegetation samples may therefore be considered "low light"-adapted. A timer-controlled pumping system connected the storage tank with a $300-\mathrm{L}$ seawater reservoir, and twice a day, the S. anglica samples were flooded for 1-h, mimicking a diurnal tidal cycle. Each of the samplecontaining aquaria had drainage holes in a vertical line $5 \mathrm{~cm}$ below the sediment surface allowing drainage of the permeable surface sediment after inundations. The vegetation samples were cultured in natural seawater diluted with tap water to a 20-ppt salinity lowering the physiological stress of salinity fluctuations caused by desiccation of the sediment during air exposure, which was observed as an important factor affecting plant health in preceding test cultures. Henceforth, all references to seawater refer to 20 -ppt seawater with a temperature of $15{ }^{\circ} \mathrm{C}$. To maintain continuous growth, the seawater was added a commercial fertilizer solution biweekly (REWE Universal flüssigdünger - a liquid NPK-fertilizer with $1.75 \%$ $\mathrm{NH}_{4}^{+}, 1.75 \% \mathrm{NO}_{3}^{-}, 3.5 \% \mathrm{CN}, 1 \%$ Guano, $5 \% \mathrm{P}_{2} \mathrm{O}_{5}$, $6 \% \mathrm{~K}_{2} \mathrm{O}$ and trace metals: $\mathrm{B}, \mathrm{Fe}, \mathrm{Mn}, \mathrm{Mo}, \mathrm{Cu}$ and $\mathrm{Zn}$ ) to a 
final concentration of $\sim 750 \mu \mathrm{mol} \mathrm{L}{ }^{-1}$ inorganic N. After 4 months, new shoots had emerged, and the vegetation samples were replanted inspecting the belowground biomass and removing dead and damaged parts and discarding unhealthy samples. The vegetation samples were cultured for four additional months and inspected again before investigations were initiated. Collection and separation of the vegetation samples inevitably cause damage to roots and rhizomes. In these experiments, the long cultivation time ( 8 months) prior to the optode studies and midway inspection of belowground biomass assured that the samples investigated had unbroken pathways from the aboveground biomass to the root tips.

\section{Preparation of Rhizosphere Studies}

The vegetation samples cultured for this experiment contained multiple shoots interconnected via rhizomes rather than single shoots. In this way, the investigated samples were representative for the plant structure in the field, where S. anglica is growing in swards, and the shoots are interconnected in dense and highly complex rhizome structures stretching more than $20 \mathrm{~cm}$ down in the sediment.

A rhizobox (Fig. 1a), an aquarium for visualization of rhizosphere processes (Neumann et al. 2009), was constructed interceding a U-shaped frame (PVC) between two transparent acrylic plastic plates making a narrow aquarium with the inner measures: h $20 \mathrm{~cm} \mathrm{w} 20 \mathrm{~cm}$ and $\mathrm{d} 2 \mathrm{~cm}$. One side of the rhizobox was glued onto the frame, while the other side was attached with 11 screw threads placed between two 3-mm neoprene round cords mounted in two 2-mm deep grooves in the frame, making the side detachable and yet water tight when attached.

To prepare the vegetation samples for planar optode studies, the rhizobox was filled with sieved sediment (mesh size $2 \mathrm{~mm}$ ) from the field by gently slurring the sediment in seawater and pouring it into the rhizobox assuring a uniform distribution without air pockets. Following, the rhizobox was submerged horizontally in a tray with seawater and the side was detached. For planar optode investigations, an oxygen sensitive optode foil was attached to the inside of the detachable side of the rhizobox using silicon grease. The $S$. anglica sample selected for investigation was gently pushed into the sediment in a position where one or more roots were lining up with the side of the rhizobox with the optode foil. The rhizobox was moved to a vertical position after reattaching the side and gently shaken, assuring a uniform distribution of the sediment around roots and rhizomes. If the roots were not aligned perfectly with the optode foil, the rhizobox was reopened, while again submerged horizontally in the tray, and the position of selected roots gently readjusted to an optimal position using tweezers. All handling of the vegetation samples in the rhizobox was performed under water preventing entrapment of air pockets inside the sediment.
The rhizobox was left undisturbed for $24 \mathrm{~h}$ reestablishing the biogeochemical conditions in the sediment.

\section{Planar Optode Studies of Rhizosphere Oxygen Dynamics}

The principle of oxygen optode investigations is based on dynamic quenching of a luminophore in the presence of oxygen (Glud et al. 1996; Holst et al. 1998). The luminophore is excited by a LED light source, and the luminescent response is recorded with a camera as luminescent lifetimes; the more oxygen present at the surface of the optode foil, the shorter the lifetime. Subsequently, each pixel in the recorded image can be ascribed an oxygen concentration value converting the recorded lifetimes to oxygen concentrations using the modified Stern-Volmer equation (Holst and Grunwald 2001). For a general introduction to the use of planar optodes for quantitative imaging in root studies, we refer to Blossfeld and Gansert (2012) and Gansert and Blossfeld (2008).

The planar optode investigations were performed using a modular luminescence lifetime imaging (MOLLI) system for imaging oxygen using luminescent transparent optode foils. The setup and principles for planar optode oxygen measurements using the MOLLI system were previously described in detail in Holst et al. (1998) and Holst and Grunwald (2001), and only the main features are outlined here. Optode foils were prepared several months in advance as described by Precht et al. (2004) coating a polyethylene terephthalate (PET) foil with a $\sim 20$ - $\mu$ m-thick sensing layer consisting of the luminophore, platinum(II) mesotetra (pentafluorophenyl) porphyrin (Frontier Scientific, Inc.), immobilized in a polystyrene matrix. The foils were stored in the dark until use. For investigations of plant-mediated sediment oxygenation, the foil was placed on the inside of the rhizobox in direct contact with the sediment and roots of the vegetation sample.

For oxygen imaging, the optode foil was excited by two blue LEDs $(\lambda \max =455 \mathrm{~nm}$ ) (LUXEON Star Royal Blue 5W LXHLMRRC), and the red luminescent light $(\lambda \max =647 \mathrm{~nm})$ emitted from the optode foil was recorded with a peltier-cooled 12 bit bw, 1,280×1,024-pixel CCD camera (SensiMod, PCO Computer Optics) equipped with a Doctor Optics Tevidon 1.6/35 lens and a red (091) filter with multi-resistant coating (MRC). To reduce noise, $2 \times 2$ pixels of the images were binned.

The CCD camera and LED light source were positioned at a distance of $\sim 20 \mathrm{~cm}$ from the rhizobox, and the pathway between the optode foil and the lens was shielded from outside light sources with a black plastic sheath allowing the luminescent response to be recorded without interference from other light sources. Data acquisition and image analysis were performed as described by Holst et al. (1998) and Holst and Grunwald (2001) using the custom-made software Look@MOLLI for lifetime imaging capturing two images during consecutive time intervals (1-11 and 12-22 $\mu \mathrm{s})$ 
after the excitation of the planar optode foil. Each optode image was recorded as an average of 16 consecutive lifetime measurements obtained within a time interval of $\sim 20 \mathrm{~s}$.

Subsequent image analysis converting luminescent lifetimes to oxygen concentration were performed with the custom-made software CalMolli v 0.93. Prior to the sediment investigations, calibration of the optode foil was performed as an area-specific three-point calibration averaging the lifetime values within a designated area of the optode foil corresponding to the area where oxygen leakage was studied. The detachable side with the optode foil was mounted on an empty rhizobox that was subsequently filled with seawater $\left(15^{\circ} \mathrm{C}\right.$, 20-ppt salinity) and bobbled with a gas mix of 100,50 and $0 \%$ atmospheric air in $\mathrm{N}_{2}$ using a precision gas mixing pump (Digamix 6KM301, Wösthoff GmbH).

\section{Selection of Vegetation Samples for Investigation}

A rhizobox with a vegetation sample representative of the in situ vegetation in regard to size, roots and rhizome structure and shoot interconnectivity was selected for investigation. The sample consisted of multiple stems $(>10)$ ranging in height from $2-30 \mathrm{~cm}$ all interconnected through rhizomes. Within the depth range of 6-10 $\mathrm{cm}$ below the sediment surface, four roots were visible in the rhizobox of which three aligned perfectly with the optode foil displaying the full oxic root zones. This rhizobox was used in the following investigations of oxic root zones in S. anglica: characterization of oxic root zones, oxygen consumption and ROL, effects of inundation and light availability, and temporal dynamics during a tidal cycle corresponding to Figs. 1, 2, 3 and 4.

Additionally, two other vegetation samples were selected for investigation. Each sample consisted of four to six stems ranging in height from 5-25 cm and had one root aligned with the optode foil at a depth of 7 and $17 \mathrm{~cm}$, respectively. In these rhizoboxes, oxic roots zones were almost fully displayed, but with minor deviations; either the root was partially covered with a layer of sand between the root and optode foil or the oxic root zone extended beyond the optode foil. These rhizoboxes were used for further investigations of effects of light availability and tidal inundation on belowground oxygen release shown in Fig. 5.

\section{Characterization of the Oxic Roots Zones in S. anglica}

In order to demonstrate the presence of oxic root zones in $S$. anglica rhizospheres, three adventitious roots were monitored simultaneously with the planar optode system. The oxygen distribution around the roots was recorded under conditions with high availability of oxygen for belowground internal transport, namely, air exposure of the aboveground biomass, assuring rapid oxygen exchange between the leaves and the surrounding atmosphere, and illumination

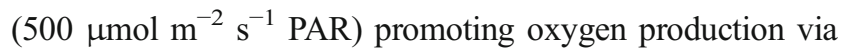
photosynthesis. Light was provided by three independent Schott KL1500 fibre optic light sources with flexible optical light guides. Henceforth, unless otherwise indicated, any reference to light availability refers to this light setting.

Investigation of Sediment Oxygen Consumption Combining the Planar Optode System with the Push-Pull Method

In order to calculate the flux of oxygen from individual roots, the specific depth sediment oxygen demand was measured directly in the rhizobox using a novel approach, combining the planar optode oxygen measurements with the push-pull technique (Koop-Jakobsen and Giblin 2009). A micropiezometer (2-mm inner diameter) connected to a peristaltic pump via PE tubing was inserted in the rhizobox sediment at $10 \mathrm{~cm}$ depth in close proximity $(2-3 \mathrm{~cm})$ of the roots investigated. Forty-millilitre pore water was extracted (pulled) from the sediment into a glass beaker at a speed of $4 \mathrm{~mL}$ per min. The extracted pore water was oxygenated with atmospheric air from an aquarium stone and injected (pushed) back into the sediment for 5-10 min reversing the flow of the peristaltic pump (Koop-Jakobsen and Giblin 2009). The plume of reoxygenated pore water was visualized in realtime via the planar optode system showing a continuously growing plume as pore water was pumped back in the sediment. Following injection, the average oxygen concentration was recorded in 30 -s intervals within a $10 \times 10 \mathrm{~mm}$ area of the optode foil located at $10 \mathrm{~cm}$ depth in the centre of the plume. The oxygen consumption rate was calculated using linear regression of the average oxygen concentration over time. At the end of the experiment, sediment porosity $(\phi)$ was measured drying five 2-mL sediment samples collected at $10 \mathrm{~cm}$ depth to constant weight. These sediment oxygen consumption measurements were conducted within $72 \mathrm{~h}$ after the root studies.

Investigation of the Effect of Light Availability and Tidal Inundation of the Aboveground Biomass on Belowground Sediment Oxygenation

The rhizobox was placed inside a tall aquaria facilitating complete submergence of the aboveground biomass with seawater. To investigate the effects of inundation and light conditions on sediment oxygenation, oxic root zones were monitored during two consecutive 12-h interval with air exposure and total submergence of the aboveground biomass, respectively. During the first $12 \mathrm{~h}$, the vegetation was airexposed, and illumination was decreased stepwise $(500,300$, 100 and $0 \mu \mathrm{mol}$ photons $\mathrm{m}^{-2} \mathrm{~s}^{-1}$ PAR) every $3 \mathrm{~h}$. Subsequently, the vegetation was completely inundated with seawater while in the dark, and the light intensity was then increased stepwise every $3 \mathrm{~h}\left(0,100,300\right.$ and $500 \mu \mathrm{mol}$ photons $\mathrm{m}^{-2} \mathrm{~s}^{-1}$ 
PAR). Oxygen images of the oxic root zones were recorded immediately before the illumination level changed. In these measurements, each pixel represented an area of $85 \times 85 \mu \mathrm{m}$. Cross-section profiles of the oxic root zones were extracted from the oxygen images, and the areas of the individual oxic root zones were calculated accounting for the number of pixels around the root with an oxygen value higher than the detection limit (defined as $3 \times$ standard deviation of an oxygen free sample; in this case, $2.3 \mu \mathrm{M}$ ).

Laboratory Investigation of the Dynamics of Oxic Root Zones During a Diurnal Tidal Cycle

The tidal dynamics of belowground sediment oxygenation was investigated, monitoring the cross-sectional area of an oxic root zone during a diurnal tidal cycle mimicked in the laboratory. The tidal cycle consisted of two consecutive 12-h periods of light and darkness, respectively, during which two 1.5-h-long tidal inundations were introduced; after 4 (in light) and $16 \mathrm{~h}$ (in dark) respectively. The aboveground biomass was completely submerged during the tidal inundations. This study was conducted $48 \mathrm{~h}$ after the previous experiment on the same vegetation sample.

Additional Investigation of the Dynamics of Oxic Root Zones in Response to Light Alternations and Inundation and in Response to Aboveground Gas Exchange with an Anoxic Atmosphere

In order to further investigate the impact of light and inundation on belowground sediment oxygenation, two additional experiments were conducted using two different vegetation samples. As the displayed roots did not align perfectly with the optode foil, maximum oxygen concentration monitored in a designated area $(1 \times 1 \mathrm{~mm})$ at the root surface was used as a proxy for oxygen release to the sediment investigating the effects of tidal inundation and changing light availability:

1. The oxygen concentration at the root surface was monitored during four consecutive 3 -h intervals of alternating light and dark conditions, 500 and $0 \mu \mathrm{mol}$ photons $\mathrm{m}^{-2} \mathrm{~s}^{-1} \mathrm{PAR}$, respectively, demonstrating the relative importance of light availability during air exposure. Subsequently, in order to demonstrate the importance of atmospheric oxygen for belowground transport and sediment oxygenation, the oxic root zone was monitored, while the whole vegetation sample was enclosed in a plastic bag and exposed to an anoxic atmosphere for $60 \mathrm{~min}$. Subsequently, the plastic bag was removed reexposing the vegetation sample to atmospheric air. The root investigated was located $17 \mathrm{~cm}$ below the sediment surface. Another type of rhizobox made from transparent polyethylene (h $25 \mathrm{~cm}, \mathrm{w} 10 \mathrm{~cm}$ and $\mathrm{d} 7 \mathrm{~cm}$ ) was used in this experiment.

2. The oxygen concentration at the root surface was monitored continuously during $4 \mathrm{~h}$ of air exposure with a stepwise hourly increase in illumination $(0,100,300$ and $500 \mu \mathrm{mol}$ photons $\left.\mathrm{m}^{-2} \mathrm{~s}^{-1} \mathrm{PAR}\right)$. Subsequently, the vegetation was completely inundated with seawater while illuminated, and the light intensity was then decreased stepwise every hour $(500,300,100$ and $0 \mu$ mol photons $\mathrm{m}^{-2} \mathrm{~s}^{-1} \mathrm{PAR}$ ) during submergence. This is the reverse procedure of the previous "light and inundation" experiment that began in light, was inundated in darkness and was followed by increasing light. The stepwise change in illumination was reduced to 1 -h intervals. The root investigated was located $7 \mathrm{~cm}$ below the sediment surface.

\section{Results}

Investigation of Oxic Root Zones in S. anglica Rhizospheres

2D images of the oxygen distribution around four roots were recorded at $7-10 \mathrm{~cm}$ depth below the sediment surface during illumination and air exposure of the aboveground biomass, clearly showing the presence of oxic zones at depth in the sediment (Fig. 1). The association between the roots and the belowground oxic zones is here illustrated merging the optode image showing the oxygen distribution and the image showing the position of the roots in the sediment. Three out of the four oxic root zones were fully visible (numbered 1-3) in the optode image, whereas one zone was only partially visible (Fig. 1b).

Oxygen leakage was restricted to root tips, with oxygen being detected only in the area immediately behind the tips, whereas no oxygen was detected further along the roots (Fig. 1b). The oxic root zones were 11.7-16.2 mm long and ranged in width from 3.3 to $4.1 \mathrm{~mm}$ measured perpendicular to the roots at the widest part of the oxic root zone. Subtracting the width of the root itself, the radius of the oxidized sediment encircling the root tips ranged from 1.3 to $1.5 \mathrm{~mm}$ measured from the root surface to the anoxic bulk sediment. The volume of the individual oxic root zones estimated as prolate spheroids ranged from 74 to $143 \mathrm{~mm}^{3}$ of which the volume of the root itself accounted for approximately $10 \%$. The oxic root zones investigated had maximum oxygen concentrations ranging from 58 to $85 \mu \mathrm{mol} \mathrm{L}^{-1}$, where the maximum oxygen concentration was measured at the root surface of each individual root and defined as the average of the five highest oxygen values detected (Table 1). 

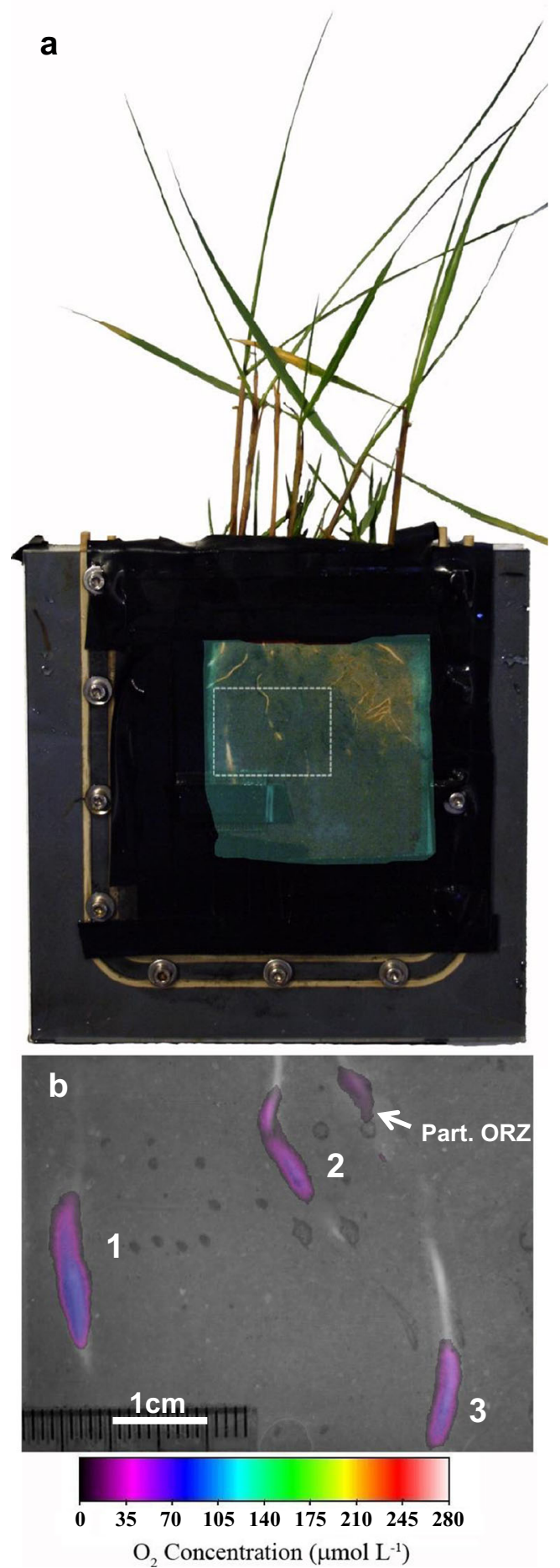

Fig. 1 Visualization of belowground oxic root zones around the root tips of Spartina anglica using planar optode technology. a Roots of S. anglica visible through the side of the rhizobox (rim of window covered with a black tape). The sediment area investigated is approximately depicted by white rectangle. b Optode image of belowground oxic root zones during air exposure in light. The image is an overlay of a transparent optode image on a bw image of the roots for visualization of the position of the oxic zones relative to the position of roots. Three fully displayed oxic root zones are numbered 1-3. A partially displayed oxic root zone is denoted "Part.ORZ".
Investigation of Sediment Oxygen Demand and Radial Oxygen Loss from Roots

Combining the push-pull method with planar optode technology, the sediment oxygen demand was measured monitoring the oxygen concentration in a plume of reoxygenated pore water injected into the sediment at $10 \mathrm{~cm}$ depth (Fig. 2). The oxygen concentration was relatively uniform in the centre of the plume at a level of approximately $130 \mu \mathrm{mol} \mathrm{L}{ }^{-1}$. All oxygen injected was consumed within a time frame of $10 \mathrm{~min}$. The average oxygen concentration in the designated area was measured over time, and the oxygen consumption rate in the pore water was calculated to $290 \mathrm{nmol} \mathrm{L}^{-1} \mathrm{~s}^{-1}$ using linear regression in the time interval from 0.5 to $7 \mathrm{~min}$, where the oxygen concentration declined linearly with time (Fig. 2b). Accounting for a sediment porosity of 0.425 , the sediment oxygen demand (SOD) per volume sediment was $123 \mathrm{nmol} \mathrm{L}^{-1} \mathrm{~s}^{-1}$.

Assuming a steady-state, where the volume of the oxic root zone is stable, the amount of oxygen removed from the root zone is counterbalanced by an equal influx of oxygen from the root. This influx was calculated based on the measured rate of sediment oxygen demand and the estimated volume of the oxic zones (excl. root) ranging from 0.009 to $0.015 \mathrm{nmol} \mathrm{s}^{-1}$. For comparison among different sediment oxidizing plants, the average radial oxygen loss across the root surface was calculated normalizing the oxygen flux with the surface area of the root estimated as being cylindrical. The radial oxygen loss per root surface area ranged from 249 to $300 \mathrm{nmol} \mathrm{m}^{-2} \mathrm{~s}^{-1}$ (Table 1).

Table 1 Standard measures and oxygen concentrations of oxic root zones

\begin{tabular}{|c|c|c|c|}
\hline & \multicolumn{3}{|c|}{ Oxic root zone no } \\
\hline & 1 & 2 & 3 \\
\hline Length (mm) & 16.2 & 12.9 & 11.7 \\
\hline Width (mm) & 4.1 & 3.3 & 3.6 \\
\hline Width of root (mm) & 1.1 & 0.8 & 0.8 \\
\hline Radius of oxic zone (mm) & 1.5 & 1.3 & 1.4 \\
\hline Max. $\mathrm{O}_{2}\left(\mu \mathrm{mol} \mathrm{L}{ }^{-1}\right)$ & 84.8 & 58.4 & 66.7 \\
\hline Cross-sectional area $\left(\mathrm{mm}^{2}\right)$ & 51.6 & 32.3 & 31.4 \\
\hline Volume $\left(\mathrm{mm}^{3}\right)$ & 143 & 74 & 80 \\
\hline Volume excl. root $\left(\mathrm{mm}^{3}\right)$ & 128 & 68 & 74 \\
\hline $\operatorname{ROL}\left(\mathrm{nmol} \mathrm{m} \mathrm{m}^{-2}\right)$ & 267 & 250 & 300 \\
\hline
\end{tabular}

The volumes of the entire oxic root zones including the roots are estimated as a prolate spheroid, and the volume of sediment oxidized is estimated subtracting the volume of the root estimated as a cylinder. The radial oxygen loss is expressed per area root surface. All other values are direct measures 


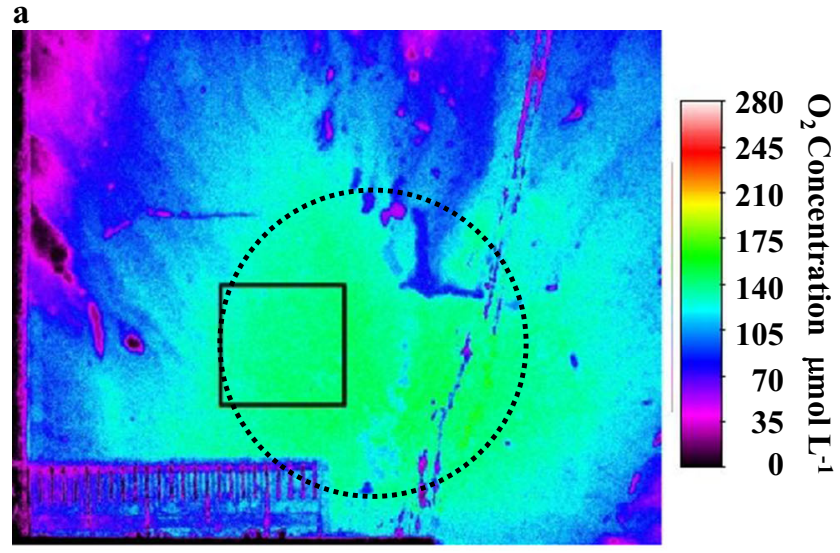

b

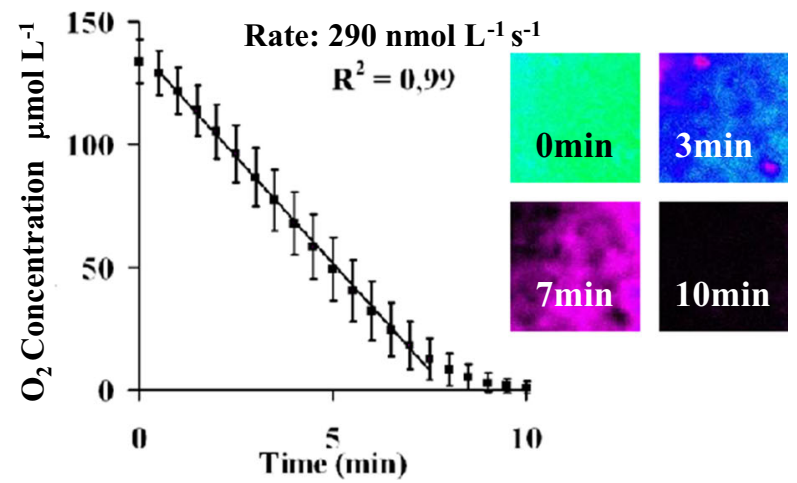

Fig. 2 Sediment oxygen demand measured combining the push-pull method and planar optode techniques. a Plume of reoxygenated pore water injected into the sediment at $10 \mathrm{~cm}$ depth. The centre of the plume had a relatively uniform oxygen concentration (punctuated circle). An area of $1 \times 1-\mathrm{cm}$ square was chosen for investigation. b Average oxygen concentration ( \pm std.) measured in the designated sediment area over time and sediment oxygen demand was calculated using linear regression. Change in the oxygen concentration over time is visualized in optode images of the selected area after $0,3,7$ and $10 \mathrm{~min}$

Investigation of Oxic Root Zones at Different Light Availabilities During Air Exposure and Inundation

During air exposure of the aboveground biomass, oxic root zones were present at all light availabilities also in complete darkness. Oxygen profiles measured perpendicular to the root at the widest part of the oxic root zones did not change in response to changing light availability, and no differences were observed neither in the width of the oxic zones nor in the maximum concentration at the root surface (Fig. 3c). Minor reductions in the cross-sectional area of the oxic root zones ranging from 8 to $29 \%$ were observed during the 12-h period of air exposure with decreasing light availability (Fig. 3b). The optode images revealed that area reduction was most pronounced at the ends of the oxic root zones (Fig. 3a), whereas the profiles (Fig. 3c) were not affected.
Therefore, the area reduction was ascribed primarily to continuous root growth/aging over time rather than changing light conditions or changing sediment conditions.

Submergence had a marked impact on plant-mediated sediment oxygenation causing a complete elimination of the oxic root zones within $3 \mathrm{~h}$ of inundation in darkness. Increasing the light availability during the following $12 \mathrm{~h}$ of inundation did not change the oxygen conditions, and sediment oxygenation remained absent during the entire inundation period.

Atmospheric oxygen was the primary source for belowground oxygen transport, and access to air was a determining factor for the degree of belowground sediment oxygenation, whereas light availability and thereby photosynthetic oxygen production only had a limited impact on the size and shape of the oxic root zones.

Investigation of the Temporal and Spatial Dynamics of an Oxic Root Zone over a 24-h Tidal Cycle

Monitoring the area of an oxic root zone during a diurnal tidal cycle showed that during a 1.5 -h tidal inundation in the light, the cross-sectional area of the oxic root zone decreased by only $36 \%$. In contrast, during inundation in the dark, the oxic root zone collapsed completely within $1 \mathrm{~h}$ (Fig. 4). Hence, during the course of a tidal cycle, photosynthetic oxygen production affected the tidal dynamics of oxic root zones in $S$. anglica rhizospheres, and the cross-sectional area of oxic zone diminished four times faster in the dark than in the light. In consequence, within the time frame of a normal tidal inundation, oxic conditions were maintained around the root tips during complete submergence in the light, whereas oxic conditions were eliminated during inundation in the dark.

This study was conducted on the same sample shown in Fig. 1, oxic root zone A, 2 days after the previous experiments. At this time point, oxic root zone $\mathrm{B}$ and $\mathrm{C}$ had diminished and were barely visible; only oxic root zone A was still clearly visible (image not shown).

Further Investigation of the Dynamics of Oxic Root Zones in Response to Aboveground Gas Exchange

During air exposure, alternating between light and dark conditions at 3-h intervals (Fig. 5a, b) showed a minor effect of light availability on oxygen release from the root. On average, the oxygen concentration at the root surface was $16 \%$ lower in the dark $\left(29.3 \pm 1.5\right.$ s.d. $\left.\mu \mathrm{mol} \mathrm{L}^{-1}\right)$ than in the light ( $35.1 \pm$ 1.5 s.d. $\mu \mathrm{mol} \mathrm{L}^{-1}$ ) comparing the average oxygen concentrations measured during light and dark intervals, respectively. The significant contribution from atmospheric oxygen to belowground sediment oxygenation was demonstrated enclosing the vegetation sample in an anoxic atmosphere 
Fig. 3 Investigation of the oxygen distribution around three roots of Spartina anglica during air exposure and complete submergence of the aboveground biomass at four different levels of light availability changing at $3-\mathrm{h}$ intervals. All images and measurements are shown in the temporal order in which they were recorded. a) Optode images of the oxygen distribution around S. anglica roots. b Area of oxic root zones recorded during changing light conditions under air exposure and inundation of the aboveground biomass, respectively, measured at the end of each light treatment. c Oxygen profiles across oxic root zones extracted from the optode images at the location represented by white punctuated line in a. Legend refers to light intensity PAR ( $\mu$ mol photons $\mathrm{m}^{-2} \mathrm{~s}^{-1}$ )
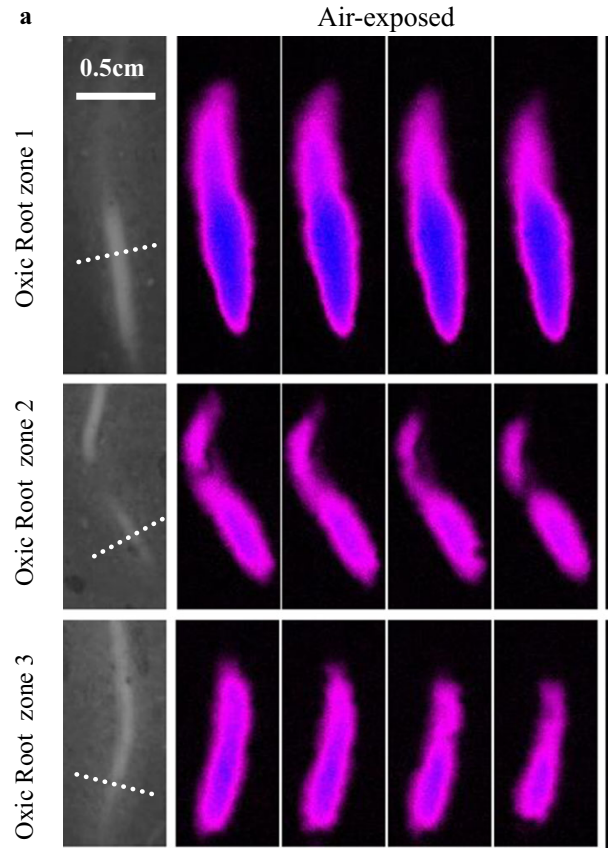

Light:

500

300

b

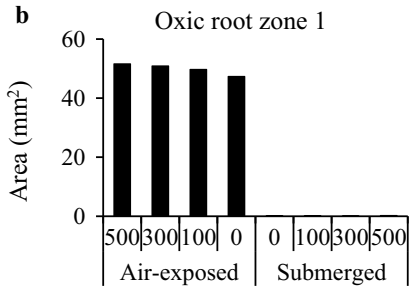

c

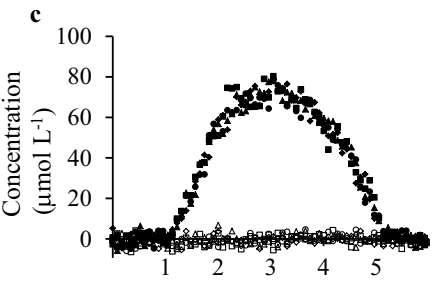

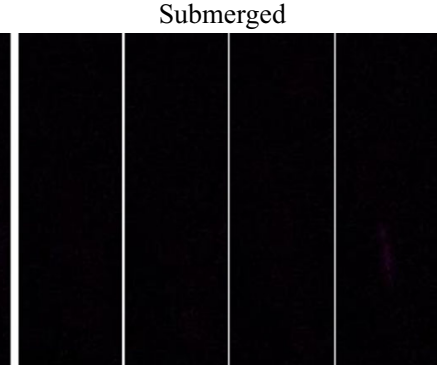
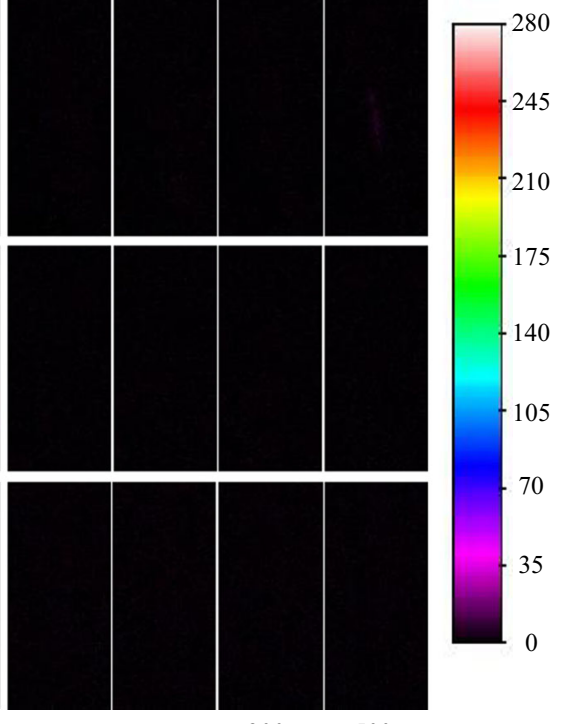

0

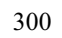

Light intensity - PAR ( $\mu$ mol photons $\left.\mathrm{m}^{-2} \mathrm{~s}^{-1}\right)$
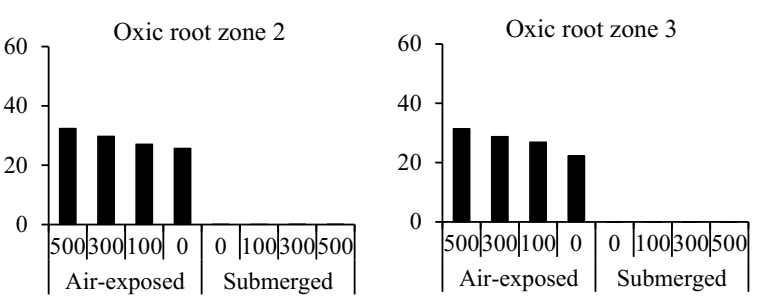

Light intensity - PAR ( $\mu$ mol photons $\mathrm{m}^{-2} \mathrm{~s}^{-1}$ )
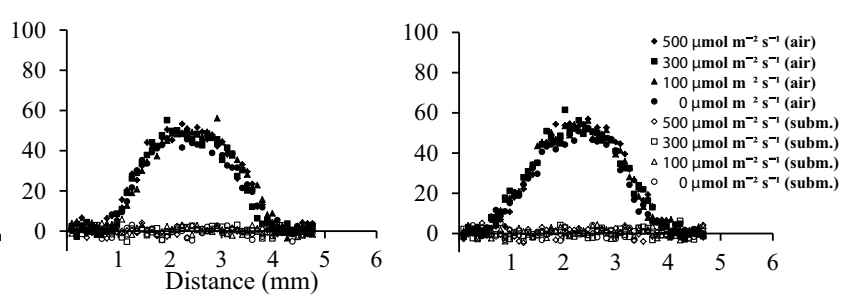

resulting in an immediate collapse of the oxic root zone within $1 \mathrm{~h}$ and instantaneous reappearance upon returning the vegetation sample to a natural atmosphere.

In the other experiment (Fig. 5c, d), during air exposure of the aboveground biomass, the oxygen concentration at the root surface increased continuously $20 \%$ from 54 in the dark to $64 \mu \mathrm{mol} \mathrm{L} \mathrm{L}^{-1}$ at the highest light level $(500 \mu \mathrm{mol}$ photons $\left.\mathrm{m}^{-2} \mathrm{~s}^{-1} \mathrm{PAR}\right)$. However, due to the steady and uninterrupted increase in the oxygen concentration over time, no direct coupling to the changing light conditions could be demonstrated.

Inundation of the aboveground biomass resulted in a significant decrease in the oxygen concentration, ultimately resulting in the termination of oxygen release in the dark.
However, during inundation, different light levels were reflected in different oxygen concentration at the root surface, which tended to level off towards a steady state concentration after $45 \mathrm{~min}$. This was observed at 300, 100 and $0 \mu \mathrm{mol}$ photons $\mathrm{m}^{-2} \mathrm{~s}^{-1}$ PAR. At $500 \mu \mathrm{mol}$ photons $\mathrm{m}^{-2} \mathrm{~s}^{-1}$, the oxygen concentration at the root surface did not level off. This was potentially caused by the aboveground biomass being fully submerged for a shorter time, since the inundation process itself lasted 20 min, during which the plants were only partially inundated. Furthermore, gas films on leaves of S. anglica may have prolonged the access to atmospheric oxygen (Winkel et al. 2011). 


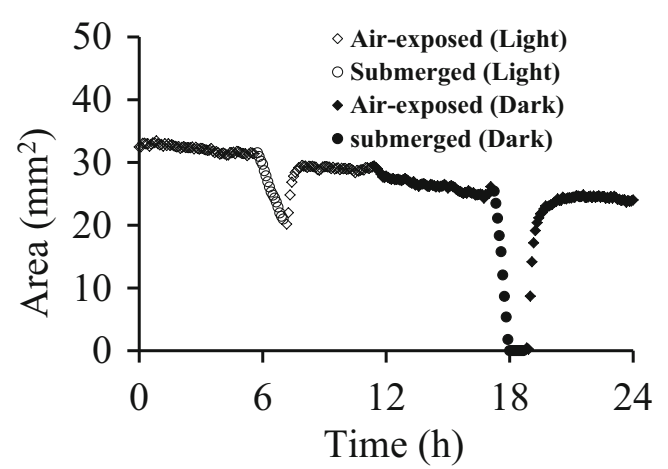

Fig. 4 Area of Spartina anglica oxic root zone monitored during a 24-h diurnal tidal cycle simulated in the laboratory. Aboveground biomass was completely inundated for $1.5 \mathrm{~h}$ at a 12 -h interval in the light and in the dark, respectively

\section{Discussion}

When belowground oxygen transport exceeds the respiratory demand of roots and rhizomes in S. anglica, oxygen can be released to the surrounding sediment. In this study, the planar optode images demonstrated the presence of distinct oxic zones around $S$. anglica roots. The planar optode images revealed that the oxic root zones were restricted to an area immediately behind root tips extending up to $16 \mathrm{~mm}$ along the root. Restriction of oxygen leakage to the tip of roots is common in other wetland plants such as common reed (Phragmites spp.) and rice (Oryza spp.), where the oxic zones
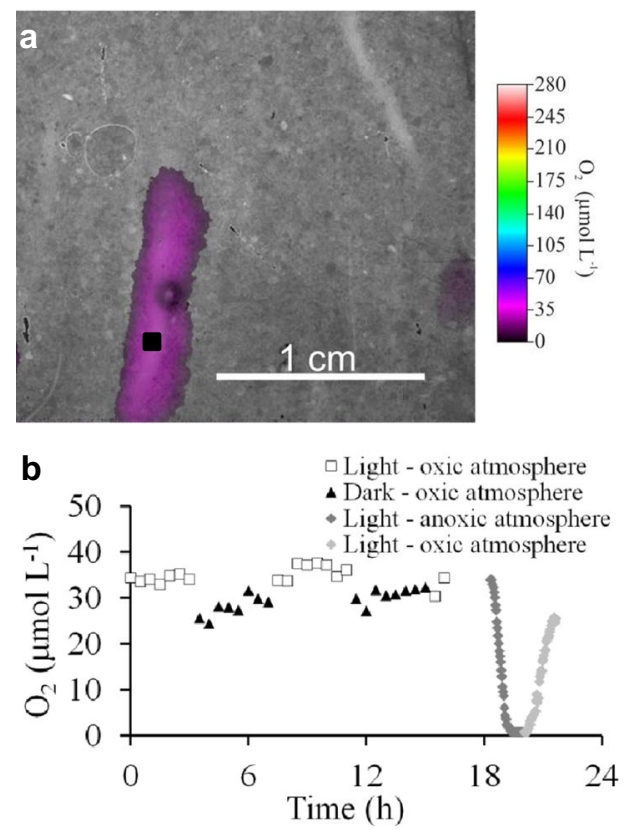

Fig. 5 Oxygen concentration measured at a root surface during alternation of light availability and access to atmospheric oxygen. a, c Optode images of the investigated oxic root zones during air exposure in light. The images are overlays of transparent optode images on bw images of the roots. Black square indicates the approximate position and area of the root surface investigated. b Oxygen concentration at the root surface typically range in length from a few millimetre to a few centimetres along the root beginning immediately behind the tips (Armstrong et al. 2000).

The width of the observed oxic root zones ranged from 1.3 to $1.5 \mathrm{~mm}$ from the root surface to the bulk anoxic sediment when monitored in light and during air exposure. This size is similar to a previous study by Holmer et al. (2002) measuring oxic zones in $S$. anglica rhizospheres using microelectrode profiling, where the widths of the oxic root zones were ranging from 1 to $2.5 \mathrm{~mm}$.

\section{Radial Oxygen Loss}

As oxygen is leaking from the roots, it is continuously consumed by microbial respiration and reoxidation of reduced compounds in the sediment. Consequently, the size of oxic root zones is directly dependent on the sediment oxygen demand. In order to calculate the diffusive flux of oxygen from the roots, obtaining depth-specific measures of oxygen consumption rates is essential (Meysman et al. 2010). The push-pull method was previously successfully applied for investigations in marsh rhizospheres (Addy et al. 2002; Koop-Jakobsen and Giblin 2010) and here in combination with planar optode technology, the combined method was very useful for determining depth-specific sediment oxygen demand inside the rhizobox (Fig. 2) with minimal disturbance
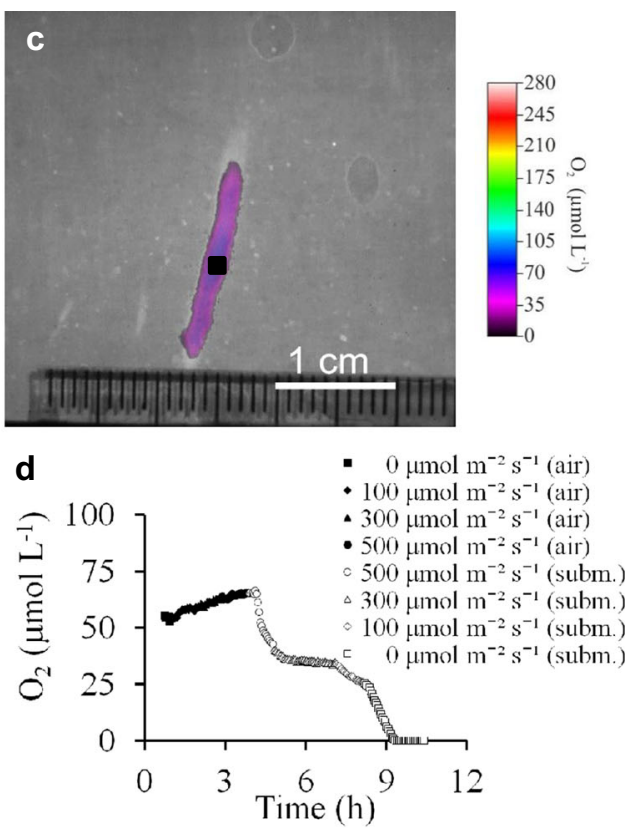

monitored during two consecutive light and dark cycles followed by exposure of the entire plant to an anoxic atmosphere $\left(100 \% \mathrm{~N}_{2}\right)$ and subsequently reintroducing a normal atmosphere. d Oxygen concentration at the root surface monitored during increasing light availability during air exposure and subsequent decreasing light availability during inundation 
of the biomass and sediment structure and thereby the microbial biota associated with sediment surfaces. The reoxidation of the extracted pore water prior to reinjection into the sediment removed some of the reduced compounds. Hence, this method primarily accounts for oxygen reduction by microbial activity, which may underestimate the total sediment oxygen demand.

The sediment oxygen demand was used to estimate the radial oxygen loss, which ranged from 250 to $300 \mathrm{nmol} \mathrm{m}^{-2} \mathrm{~s}^{-1}$. Lai et al. (2012) investigated 35 wetland plants primarily from freshwater habitats and found the highest oxygen flux from the roots to be $786 \mathrm{nmol} \mathrm{m}^{-2} \mathrm{~s}^{-1}$ (Caldesia reniformis) and $85 \%$ of the investigated species being above $260 \mathrm{nmol} \mathrm{m}^{-2} \mathrm{~s}^{-1}$. Colmer (2003) summarized radial oxygen loss from five different wetland species specifically focussing on the area $5 \mathrm{~mm}$ behind the root tips and found the oxygen fluxes ranging from 45 to $390 \mathrm{nmol} \mathrm{m}^{-2} \mathrm{~s}^{-1}$ with Phragmites australis representing the highest value. The radial oxygen loss of $S$. anglica measured in these studies is in the same order of magnitude as other common wetland plants, and the high capability for oxygen transport through the plant observed in other studies (Maricle and Lee 2007) is here not reflected in the recorded sediment oxygenation capacity.

The planar optode technique is an excellent tool for visualizing oxygen dynamics in sediments; however, for radial oxygen loss measurements, quantification should be done with caution (Glud 2008). Since the side of the rhizobox acts as an impermeable barrier, oxygen accumulates in front of the optode foil enlarging the plane of the oxic plume (Polerecky et al. 2006). Based on a modelling approach, Frederiksen and Glud (2006) suggested that planar optode measurements overestimated the radius of oxic root zones of $Z$. marina by a factor of 1.4. As the oxic root zones of $S$. anglica investigated here are similar in size and geometry, we expect that a similar degree of overestimation may apply in our studies. Despite this systematic drawback of the planar optode technique, we still find it valid to determine whether the sediment oxygenation in $S$. anglica is markedly different from other wetland species. This was however not the case.

\section{The Effects of Light Availability During Air Exposure}

During air exposure, light availability only had a minor effect on belowground sediment oxygenation, showing that atmospheric oxygen is the primary source during air exposure. The direct connection and potential for rapid gas exchange between the atmosphere and the belowground oxic zones were demonstrated placing an entire plant in an anoxic atmosphere $\left(100 \% \mathrm{~N}_{2}\right)$, resulting in an immediate collapse of the oxic root zone within $1 \mathrm{~h}$ and instantaneous reappearance upon returning the plant to a natural atmosphere (Fig. 5b).

Different experiments responded differently to changes in light availability, but in general, the effect of light availability on belowground sediment oxygenation was minor during air exposure. One vegetation sample showed no effect on oxygen profiles across the oxic root zone and oxygen concentrations at the root surface in response to decreasing light availability (Fig. 3c). In contrast, two different vegetation samples showed a 16 and $20 \%$ difference in the oxygen concentration at the root surface between dark and light conditions during two light/dark cycles (Fig. 5b) and during a stepwise increase in light availability (Fig. 5d), respectively.

The magnitude of the effect of light availability shown here is comparable with observations by Winkel et al. (2011) finding that the internal oxygen concentrations inside the rhizomes of $S$. anglica declined $23-33 \%$ in the dark. In contrast, studies by Holmer et al. (2002) found the oxygen concentration at the root surface of S. anglica to be $80 \%$ lower in darkness. We presume that this marked divergence among different studies can be ascribed to marked differences in the aboveground biomass of $S$. anglica. In our study and in the study by Winkel et al. (2011), the shoot heights were no more than $40 \mathrm{~cm}$, whereas the shoot height was up to $80 \mathrm{~cm}$ in the study by Holmer et al. (2002). Previous studies have demonstrated that short forms of the closely related S. alterniflora exhibited a lower specific gas transport capacity than taller forms of S. alterniflora plants (Arenovski and Howes 1992).

The Effects of Inundation and Light Availability During Inundation

Inundation of the aboveground biomass preventing access to atmospheric air resulted in a significant reduction of belowground sediment oxygenation and the distribution of oxygen around the root tips (Figs. 3, 4 and 5). These observations are concurrent with studies investigating the internal oxygen concentrations inside rhizomes of $S$. anglica (Winkel et al. 2011) and inside the shoot base of $S$. alterniflora (Gleason and Zieman 1981), demonstrating that rhizome oxygen concentrations dropped significantly during inundation.

The effect of light on sediment oxygenation during inundation was dependant on whether the inundation occurred in light or in darkness. Total submergence in the dark always resulted in the termination of sediment oxygenation and a complete collapse of the oxic root zones (Figs. 3, 4 and 5), showing that the surrounding water was an insufficient oxygen source for belowground sediment oxygenation. Under these conditions, the collapse of the oxic root zones could happen rapidly within $1 \mathrm{~h}$ of inundation (Fig. 4). A step-wise increase in light availability during prolonged inundation ( $>3 \mathrm{~h}$ ) after the oxic root zone had collapsed did not reestablished significant sediment oxygenation (Fig. 3). During prolonged flooding with anoxic sediment conditions, reduced compounds may built up in the previously oxygenated zones preventing a rapid reoccurrence of oxic root zones at higher light intensities. 
During inundation in the light, $S$. anglica was able to sustain sediment oxygenation even during prolonged flooding $(>3 \mathrm{~h})$ and at low light availability $\left(100 \mu \mathrm{mol}\right.$ photons $\mathrm{m}^{-2} \mathrm{~s}^{-1}$ PAR), however, at significantly reduced levels in comparison to air-exposed conditions (Fig. 5d).

\section{Sediment Oxygenation in a Diurnal Tidal Cycle Perspective}

When the effects of tidal inundations were investigated over a laboratory-simulated tidal cycle with inundations lasting $1.5 \mathrm{~h}$ (Fig. 4), photosynthetically produced oxygen did affect the sediment oxygenation dynamics. Here, the cross-sectional area of the monitored oxic root zone decreased continuously to $68 \%$ of its original size after $1.5 \mathrm{~h}$ inundation in the light, whereas the entire oxic root zone was eliminated within $1 \mathrm{~h}$ of inundation in the dark. These results show that within the time frame of a normal tidal inundation, the belowground sediment oxygenation is less affected by tidal inundations in the light than in darkness.

For $S$. anglica growing in sandy permeable sediments along the Wadden Sea coastline, tidal inundation usually lasts 1-2 $\mathrm{h}$, and light may therefore contribute to the maintenance of oxic root zones in the rhizosphere during tidal submergence in the daytime. However, in a diurnal tidal system, the rhizosphere of $S$. anglica will inevitably also frequently experience periods with no sediment oxygenation during inundation in darkness.

Located in the low tidal marsh, S. anglica is exposed to frequent tidal inundations of variable length depending on the neap-spring tidal cycle and local weather conditions. At the location, where the vegetation samples for these studies were collected, periods with prolonged onshore winds can cause inundation of the vegetation for extended time periods around high tide lasting more than $3 \mathrm{~h}$ (personal observation). For this situation, our results suggest that $S$. anglica will be able to sustain sediment oxygenation during prolonged inundation in the light (Fig. 5d). However, if prolonged tidal inundations occur at night, sediment oxygenation is terminated and oxic root zones collapse. Even if the prolonged inundation lasts until daylight, the sediment will remain anoxic until the vegetation is exposed to atmospheric air (Fig. 3). Even so, $S$. anglica is highly tolerant to inundation and thrive even under conditions with 10-h daily immersion ( $\mathrm{Li}$ et al. 2011). Also in the field, $S$. anglica was observed growing in areas exposed to occasional 11-h continuous inundation (Hubbard 1969).

Interferences from Root Growth/Aging and Reduced Sediment Compounds

The cross-sectional area of the oxic roots zones decreased by up to $29 \%$ during the $12 \mathrm{~h}$ of air exposure with stepwise decreasing light availability (Fig. 3b). This reduction was, however, not reflected in the oxygen profiles measured at the widest part of the oxic root zone or in the maximum oxygen concentration at the root surface (Fig. 3c). The corresponding optode images (Fig. 3a) indicated that area reduction was most pronounced at the ends of the oxic zone. Consequently, factors other than decreasing light availability, such as continuous growth and aging of roots, are expected to have caused the decline in the cross-sectional areas. In our studies, an elongation of the roots was not visible in the optode images, but in the experiment monitoring an oxic root zone over time during a laboratory-simulated tidal cycle (Fig. 4), it was clearly demonstrated that the cross-sectional area decreased continuously over time during air exposure in the light as well as in the dark.

A continuous build-up of reduced compounds in the sediment would also contribute to a decline in the cross-sectional area of the oxic zone. However, since the area reduction was most pronounced at the ends of the oxic zone and not reflected in the width, the observed change in cross-sectional area is ascribed to aging of the roots changing the oxygen permeability over time. Root growth and aging of roots were previously observed as a factor affecting root oxygen-leaking properties over time in other studies of belowground oxygen dynamics using planar optodes (Frederiksen and Glud 2006; Jensen et al. 2005)

\section{Future Research}

In this study, we demonstrated the presence of oxic zones around S. anglica roots at depth in the sediment and revealed how the plant-mediated sediment oxygenation is dynamic and fluctuate with tidal inundations and changing light conditions. The studies were conducted on relatively small plants growing in permeable sandy sediments. Around the world, S. anglica thrive in a wide variety of substrates, ranging from clays over fine silts and organic muds to sands and shingle, and exhibits highly variable morphology with large differences in shoot height and biomass (Gray et al. 1991; Van Hulzen et al. 2007). Sediment oxygenation dynamics may vary markedly among different ecotypes of $S$. anglica and the redox characteristics of the sediment it inhabits. Further studies are needed in order to describe the belowground sediment oxygenation in different habitats and account for the role of shoot morphology and substrate.

Acknowledgments This research was funded by SENSEnet, a Marie Curie Initial Training Network funded by the European Commission Seventh Framework Programme (PITN-GA-2009-237868), and by funding received from the Leibniz Program of the DFG to Prof. Dr. Antje Boetius. Additionally, the research was funded through the Max Planck Society. The custom-made software programs used for data acquisition and analysis were developed by Björn Grünwald (Calmolli v.0.93) and Björn Grünwald and Lubos Polerecky (Look@MOLLI server v2.01).We 
thank the Alfred Wegener Institute, Wadden Sea Station, Sylt, for assistance with fieldwork.

Open Access This article is distributed under the terms of the Creative Commons Attribution License which permits any use, distribution, and reproduction in any medium, provided the original author(s) and the source are credited.

\section{References}

Addy, K., D.Q. Kellogg, A.J. Gold, P.M. Groffman, G. Ferendo, and C. Sawyer. 2002. In situ push-pull method to determine ground water denitrification in riparian zones. Journal of Environmental Quality 31: $1017-1024$

Arenovski, A.L., and B.L. Howes. 1992. Lacunal allocation and gas transport capacity in the salt marsh grass Spartina alterniflora. Oecologia 90: 316-322.

Armstrong, W., and E.J. Wright. 1975. Radial oxygen loss from rootsTheoretical basis for manipulation of flux data obtained by cylindrical platinum-electrode technique. Physiologia Plantarum 35: 21-26.

Armstrong, W., D. Cousins, J. Armstrong, D.W. Turner, and P.M. Beckett. 2000. Oxygen distribution in wetland plant roots and permeability barriers to gas-exchange with the rhizosphere: A microelectrode and modelling study with Phragmites australis. Annals of Botany 86: 687-703.

Blossfeld, S. 2013. Light for the dark side of plant life: Planar optodes visualizing rhizosphere processes. Plant and Soil 369: 29-32.

Blossfeld, S., and D. Gansert. 2012. The use of planar optodes in root studies for quantitative imaging. In Measuring roots: An updated approach, ed. S. Mancuso, 83-92. Berlin Heidelberg: Springer.

Blossfeld, S., D. Gansert, B. Thiele, A.J. Kuhn, and R. Loesch. 2011. The dynamics of oxygen concentration, $\mathrm{pH}$ value, and organic acids in the rhizosphere of Juncus spp. Soil Biology and Biochemistry 43: 1186-1197.

Bradley, P.M., and J.T. Morris. 1990. Influence of oxygen and sulfide concentration on nitrogen uptake kinetics in Spartina alterniflora. Ecology 71: 282-287.

Brix, H. 1994. Functions of macrophytes in constructed wetlands. Water Science and Technology 29: 71-78.

Colmer, T.D. 2003. Long-distance transport of gases in plants: A perspective on internal aeration and radial oxygen loss from roots. Plant, Cell and Environment 26: 17-36.

Frederiksen, M.S., and R.N. Glud. 2006. Oxygen dynamics in the rhizosphere of Zostera marina: A two-dimensional planar optode study. Limnology and Oceanography 51: 1072-1083.

Gansert, D., and S. Blossfeld. 2008. The application of novel optical sensors (optodes) in experimental plant ecology. In Progress in botany, ed. U. Lüttge, W. Beyschlag, and J. Murata, 333-358. Berlin Heidelberg: Springer.

Giblin, A.E., and R.W. Howarth. 1984. Porewater evidence for a dynamic sedimentary iron cycle in salt marshes. Limnology and Oceanography 29: 47-63.

Gleason, M.L., and J.C. Zieman. 1981. Influence of tidal inundation on internal oxygen supply of Spartina alterniflora and Spartina patens. Estuarine, Coastal and Shelf Science 13: 47-57.

Glud, R.N. 2008. Oxygen dynamics of marine sediments. Marine Biology Research 4: 243-289.

Glud, R.N., N.B. Ramsing, J.K. Gundersen, and I. Klimant. 1996. Planar optrodes: A new tool for fine scale measurements of twodimensional $\mathrm{O}-2$ distribution in benthic communities. Marine Ecology-Progress Series 140: 217-226.

Gray, A.D., Marshall., and A. Raybould. 1991. A century of evolution in Spartina anglica. In Advances in ecological research, Begon, M.,
A.H. Fitter, and A. Macfadyen. 21: 1-62. London Academic Press. doi:10.1016/S0065-2504(08)60096-3.

Holmer, M., B. Gribsholt, and E. Kristensen. 2002. Effects of sea level rise on growth of Spartina anglica and oxygen dynamics in rhizosphere and salt marsh sediments. Marine Ecology-Progress Series 225: 197-204.

Holst, G., and B. Grunwald. 2001. Luminescence lifetime imaging with transparent oxygen optodes. Sensors and Actuators B: Chemical 74: 78-90.

Holst, G., O. Kohls, I. Klimant, B. Konig, M. Kuhl, and T. Richter. 1998. A modular luminescence lifetime imaging system for mapping oxygen distribution in biological samples. Sensors and Actuators B: Chemical 51: 163-170.

Howes, B.L., and J.M. Teal. 1994. Oxygen loss from Spartina alterniflora and its relationship to salt marsh oxygen balance. Oecologia 97: 431-438.

Hubbard, J.C.E. 1969. Light in relation to tidal immersion and the growth of Spartina townsendii (s.1.). Journal of Ecology 57: 795-804.

Jensen, S.I., M. Kuhl, R.N. Glud, L.B. Jorgensen, and A. Prieme. 2005. Oxic microzones and radial oxygen loss from roots of Zostera marina. Marine Ecology-Progress Series 293: 49-58.

Koop-Jakobsen, K., and A.E. Giblin. 2009. New approach for measuring denitrification in the rhizosphere of vegetated marsh sediments. Limnology and Oceanography: Methods 7: 626-637.

Koop-Jakobsen, K., and A.E. Giblin. 2010. The effect of increased nitrate loading on nitrate reduction via denitrification and DNRA in salt marsh sediments. Limnology and Oceanography 55: 789-802.

Lai, W.-L., Y. Zhang, and Z.-H. Chen. 2012. Radial oxygen loss, photosynthesis, and nutrient removal of 35 wetland plants. Ecological Engineering 39: 24-30.

Lee, R.W. 1999. Oxidation of sulfide by Spartina alterniflora roots. Limnology and Oceanography 44: 1155-1159.

Lee, R.W. 2003. Physiological adaptations of the invasive cordgrass Spartina anglica to reducing sediments: Rhizome metabolic gas fluxes and enhanced O-2 and H2S transport. Marine Biology 143: $9-15$

Li, H., G. Lei, Y. Zhi, P. Bridgewater, L. Zhao, Y. Wang, Z. Deng, Y. Liu, F. Liu, and S. An. 2011. Phenotypic responses of Spartina anglica to duration of tidal immersion. Ecological Research 26: 395-402.

Maricle, B.R., and R.W. Lee. 2002. Aerenchyma development and oxygen transport in the estuarine cordgrasses Spartina alterniflora and S. anglica. Aquatic Botany 74: 109-120.

Maricle, B.R., and R.W. Lee. 2007. Root respiration and oxygen flux in salt marsh grasses from different elevational zones. Marine Biology 151: 413-423.

Mendelssohn, I.A., and M.T. Postek. 1982. Elemental analysis of deposits on the roots of Spartina alterniflora Loisel. American Journal of Botany 69: 904-912.

Meysman, F.J.R., O.S. Galaktionov, R.N. Glud, and J.J. Middelburg. 2010. Oxygen penetration around burrows and roots in aquatic sediments. Journal of Marine Research 68: 309-336.

Minett, D.A., P.L.M. Cook, A.J. Kessler, and T.R. Cavagnaro. 2013. Root effects on the spatial and temporal dynamics of oxygen in sandbased laboratory-scale constructed biofilters. Ecological Engineering 58: 414-422.

Nehring, S., and H. Adsersen. 2006. NOBANIS - Invasive alien species fact sheet-Spartina anglica: From: online database of the North European and Baltic Network on Invasive Alien Species-NOBANIS www.nobanis.org, date of access 15.03.2014.

Nehring, S., and K.-J. Hesse. 2008. Invasive alien plants in marine protected areas: The Spartina anglica affair in the European Wadden Sea. Biological Invasions 10: 937-950.

Neumann, G., T. George, and C. Plassard. 2009. Strategies and methods for studying the rhizosphere-The plant science toolbox. Plant and Soil 321: 431-456. 
Pezeshki, S.R. 2001. Wetland plant responses to soil flooding. Environmental and Experimental Botany 46: 299-312.

Polerecky, L., N. Volkenborn, and P. Stief. 2006. High temporal resolution oxygen imaging in bioirrigated sediments. Environmental Science \& Technology 40: 5763-5769.

Precht, E., U. Franke, L. Polerecky, and M. Huettel. 2004. Oxygen dynamics in permeable sediments with wave-driven pore water exchange. Limnology and Oceanography 49: 693-705.

Sand-Jensen, K., C. Prahl, and H. Stokholm. 1982. Oxygen release from roots of submerged aquatic macrophytes. Oikos 38: 349-354.
Teal, J.M., and J.W. Kanwisher. 1966. Gas transport in the marsh grass Spartina alterniflora. Journal of Experimental Botany 17: 355-361.

Van Hulzen, J.B., J. Van Soelen, and T.J. Bouma. 2007. Morphological variation and habitat modification are strongly correlated for the autogenic ecosystem engineer Spartina anglica (Common cordgrass). Estuaries and Coasts 30: 3-11.

Winkel, A., T.D. Colmer, and O. Pedersen. 2011. Leaf gas films of Spartina anglica enhance rhizome and root oxygen during tidal submergence. Plant, Cell \& Environment 34: 20832092. 